\title{
Spectrum Sensing and Resource Allocation Models for Enhanced OFDM Based Cognitive Radio
}

\author{
Sener Dikmese, AlaaEddin Loulou, Sudharsan Srinivasan and Markku Renfors \\ Department of Electronics and Communications Engineering \\ Tampere University of Technology \\ Tampere, Finland \\ \{sener.dikmese, alaa.loulou, sudharsan.srinivasan, markku.renfors\}@tut.fi
}

\begin{abstract}
Multicarrier waveforms have been commonly proposed as strong candidates for cognitive radio (CR) due to their high spectrum efficiency, flexibility in resource allocation and commonality of signal processing for spectrum sensing and spectrum utilization. OFDM based 802.11 Wireless Local Area Network (WLAN) technologies are good candidates as CR waveforms. However, the OFDM based systems have significant limitations as CR due to their limited spectral containment, which degrades the performance in determining the free spaces and in coordinating the spectrum usage. Therefore, methods for suppressing the spectral sidelobes of OFDM are considered in this study. In particular, a combination of edge windowing and cancellation carrier techniques is applied for sidelobe suppression. In this study, such an enhanced OFDM scheme is compared with traditional OFDM and filter bank based schemes in spectrum sensing and spectrum allocation, considering also the spectral leakage effects appearing in practical WLAN devices due to power amplifier nonlinearity.
\end{abstract}

Index Terms - cognitive radio, FFT, filter bank, enhanced OFDM, edge windowing, cancellation carrier, spectrum sensing, spectrum utilization, loading algorithms.

\section{INTRODUCTION}

The usage of wireless communication devices is growing exponentially and they are widely produced all around the world. One of the important challenges in maximizing the efficiency of the spectrum use is to improve the interference control between different systems/users [1] . Especially, the $2.4 \mathrm{GHz}$ ISM band is freely available and hence used by various kinds of wireless systems due to the global availability. To reduce interference and better utilize the spectrum, cognitive radio (CR) and advanced signal processing techniques for efficient spectrum use have been studied extensively [2], [3], [4] and [5].

Spectrum sensing is an important part of CR applications. It is used for identifying spectrum holes in an efficient way. With reliable spectrum information, a CR can provide a noninterfered reliable communication. Repeated monitoring and cooperation with other users is necessary to obtain reliable spectrum information due to varying channel conditions and radio scene [6].
Most of the recent wireless communication standards have preferred to use cyclic prefix based CP-OFDM techniques due to its reliable and robust performance and simplicity of the signal processing functions on the transmitter and receiver sides. CP-OFDM is the most well-known multicarrier technology, since it is adopted in many popular standards, e.g., WiMAX, 3GPP LTE, 802.11a/g/n, and DVB. However, also alternative multicarrier techniques have been studied increasingly in the literature to get rid of spectral leakage which comes from the sidelobes. Especially, enhanced OFDM based techniques have been realized to have various potential benefits in the CR context. Hence, various enhancements are suggested in the literature improving the spectral efficiency of OFDM scheme. An interesting enhancement for OFDM model is the combination of edge windowing technique and cancellation carrier techniques [7], [8], [9], [10] and [11].

Wideband energy detector based multichannel spectrum sensing techniques are considered in this paper. By averaging the output samples of a filter bank based spectrum analyzer simultaneously for multiple center frequencies and bandwidths, multiple spectral gaps can be tested and identified rapidly in an efficient and flexible way.

As a second step, efficient spectrum utilization is important in maximizing the cognitive radio's throughput after the spectrum sensing process. The performance of the spectrum utilization can be improved with proper loading algorithms [12], [13] and [14]. Loading algorithms require knowledge of the channel. The channel state information (CSI) gives this information to the transmitter when there is a feedback from the receiver. The transmit power and/or the data rate can be adapted at the transmitter according to the CSI. The adaptation algorithms use commonly the waterfilling principle. The water-filling solution can be thought of as the curve of inverted channel signal to noise ratio (SNR) being filled with energy to a constant line. There are two different variations of these algorithms, rate adaptive and margin adaptive [15]. While using these algorithms, it is common to assume that the channel is quasi-static. Therefore once the allocation of bits and energy is done at the beginning 
of the transmission, it can be maintained until a new set of CSI is made available.

Here, we consider maximizing the data rate of a $\mathrm{CR}$ operating in a spectral hole. The secondary transmission power is controlled adaptively. Hence, the interference towards the primary is kept to minimum. To meet this goal, we choose the rate adaptive algorithm in this study. The total data rate of the $\mathrm{CR}$ is maximized when the achievable rate for each subband is maximized constraint on the total energy that is allowed for the CR transmission symbols without causing any substantial increase in the interference [15].

In Section 2, traditional and Enhanced OFDM based WLAN signal models are given. FFT and analysis filter bank (AFB) based spectrum sensing is reviewed, considering the spectrum analysis aspects related to the multicarrier techniques in Section 3. An efficient spectrum utilization model will be presented in Section 4. Section 5 presents the simulation results for the considered radio scene. Finally, some concluding remarks are presented about the performance of these methods.

\section{TRADITIONAL AND ENHANCED OFDM BASED WLAN SIGNAL MODELS}

Even though CP-OFDM is the most well-known multicarrier technology, enhanced OFDM based WLAN signal model can be used to overcome the spectral leakage problems. Basically, each OFDM subcarrier is expressed mathematically by a sinc function due to the rectangular shape of the CP-OFDM symbol in time domain. Accordingly, the spectrum of the OFDM symbol can be expressed as follows

$$
S(f)=T_{s} \sum_{k=0}^{N_{\text {IFFT }}-1} x_{k} \operatorname{sinc}\left[T_{s}\left(f-\frac{k}{T_{u}}\right)\right]
$$

where $\left\{x_{0}, x_{1}, \ldots x_{N_{I F F T}-1}\right\}$ are the input samples to the IFFT with the block of size $N_{I F F T}, T_{u}$ is the useful symbol duration, $T_{s}$ is the total CP-OFDM symbol duration and $\sin \mathrm{c}=\sin (\pi x) / \pi x$.

To improve the spectral efficiency of OFDM scheme, a combination of two techniques is used. The combination utilizes the edge windowing scheme, which is a special form of time domain windowing [8]. Edge windowing divides the subcarriers in several groups, usually two: edge group and inner group. The edge group contains the subcarriers around the edges where long window and short CP are utilized. Other subcarriers are contained in the inner group, where short window and long CP are applied. This technique introduces a controllable Intercarrier Interference/Intersymbol Interference (ICI/ISI), while providing efficient sidelobes suppression. Edge windowing can be combined with the partial transmit sequence (PTS) for peak to average power ratio (PAPR) mitigation in computationally efficient way [11]. However, time domain windowing technique has insufficient suppression performance on sidelobes close to active subcarriers [7]. Hence, a simplified version of the cancellation carrier scheme is used in the combination since cancellation carrier technique has effective suppression performance on sidelobes near the edges [9]. The simplified cancellation carrier model reduces the computational complexity significantly compared to the common cancellation carrier scheme, especially in non-contiguous scenarios and power limited cases [10]. The reduction in complexity is produced by optimizing each edge separately. If the number of used cancellation carrier on each edge is $V$ and the number of optimization points is $U$, the optimization problem is expressed in the following way

$$
\min _{z}\|\mathbf{Q}+\mathbf{C z}\|
$$

where $\mathbf{Q}=\left[Q_{1}, Q_{2}, \ldots, Q_{U}\right]^{T}$ the sidelobes values at the chosen optimization points and $\mathbf{C}=\left[C_{1}, C_{2}, \ldots, C_{V}\right]$ contains the sidelobes values of the inserted cancellation carriers in the optimization points. Moreover, the column vector $\mathbf{C}_{1}=\left[C_{11}, C_{21}, \ldots, C_{U 1}\right]^{T}$ represents the sidelobes values of the un-weighted cancellation carrier at the optimization points, and $\mathbf{z}=\left[z_{1}, z_{2}, \ldots, z_{V}\right]^{T}$ are the cancellation carriers' weights. The solution of the problem in (2) is then [16]

$$
\mathbf{z}=-\mathbf{C}^{\dagger} \mathbf{P}
$$

where $\mathbf{C}^{\dagger}=\left(\mathbf{C}^{\mathbf{T}} \mathbf{C}\right)^{-1} \mathbf{C}^{\mathbf{T}}$ is the pseudo-inverse of the matrix C. In figure 1 , the implementation of the combination is illustrated. The first block of the implementation evaluates the weight vector $\mathbf{z}$. It is critical to consider the edge windowing effect in the evaluation of the matrices $\mathbf{P}$ and $\mathbf{C}$. Otherwise, the cancellation carrier technique will be inefficient. Regarding edge windowing, two IFFTs and two CP\&window blocks are required. The division allows applying different $\mathrm{CP}$ and window lengths on the two groups. The sum of the edge group branch and the inner group branches results in the required enhanced CP-OFDM signal.

The combination results in strong suppression of the sidelobes since the cancellation carrier targets the close subcarriers and edge windowing suppresses the farther subcarriers. Nevertheless, the combination needs an extension to CP-OFDM symbol in order to apply time windowing. To reach sufficient sidelobe suppression performance, we use two cancellation carriers at both edges, increasing the total number of transmitted subcarriers by four.

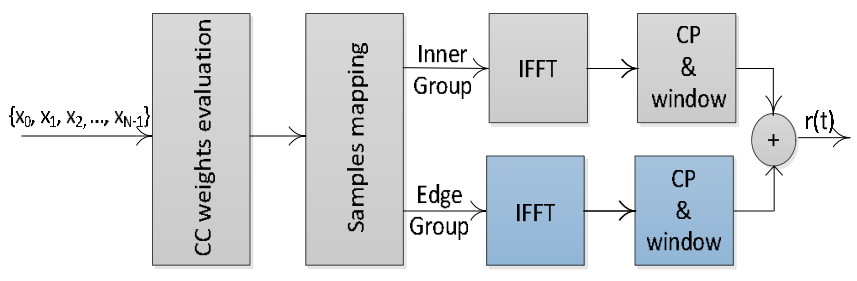

Figure 1. Block diagram of enhanced OFDM signal 
In our numerical studies, we consider a scenario with two active $802.11 \mathrm{~g}$ type traditional or enhanced OFDM based WLANs signals with similar parameters, as shown in figure 2. The two channels are assumed to have the same power level, normalized to $0 \mathrm{~dB}$. In this case, the traditional and enhanced OFDM based WLAN1 and WLAN2 signals use the channels 3 and 8 , out of the entire 11 different channels. The channels don't overlap and there is $8 \mathrm{MHz}$ spectrum hole available in this scenario. Due to the transmitter power amplifier (PA) non-linearity, spectral regrowth gets introduced, raising the spectral density in the nearby frequencies. Considering the worst case situation allowed by the $802.11 \mathrm{~g}$ specifications, the power spectrum density in the gap between the two channels can be at about $-20 \mathrm{dBr}(20 \mathrm{~dB}$ below the pass-band level) [4]. For realistic model of the power amplifier effects, we use the Rapp model [17]. The mentioned worst-case situation corresponds to $5 \mathrm{~dB}$ back-off. We consider also $15 \mathrm{~dB}$ back-off for modest spectrum regrowth, and also the ideal power amplifier case is included as a reference. The specific enhanced OFDM based WLAN design has at least $40 \mathrm{~dB}$ stop band attenuation. However, depending on the linearity of the PA, some spectrum leakage would be present also in the enhanced OFDM based WLAN case.

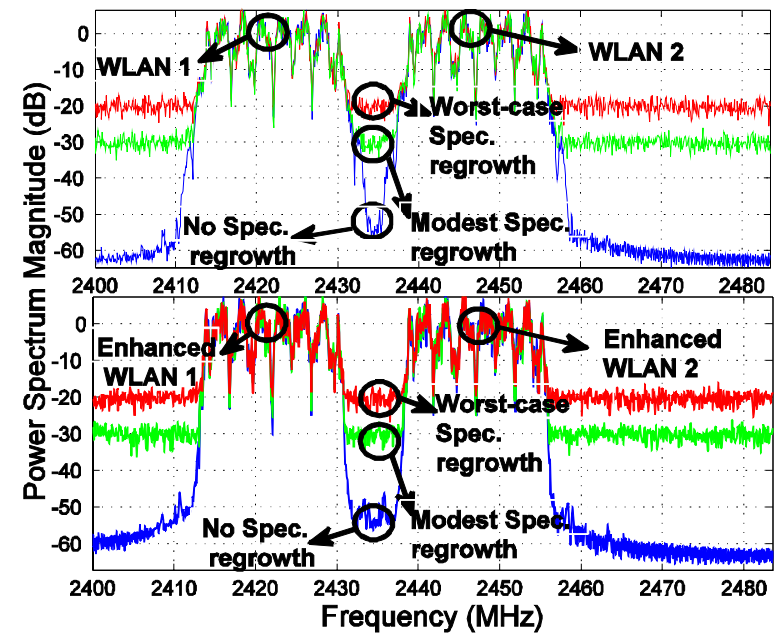

Figure 2. Two traditional and enhanced WLAN signals using 3rd and 8th WLAN channels in the $2.4 \mathrm{GHz}$ ISM band.

\section{FFT AND AFB BASED SPECTRUM SENSING}

The schemes of alternative FFT and AFB based spectrum sensing algorithms are illustrated in figure 3 .
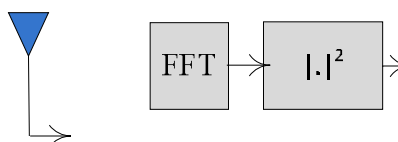

Time and Frequency

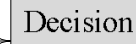

WLAN

\&Enh.
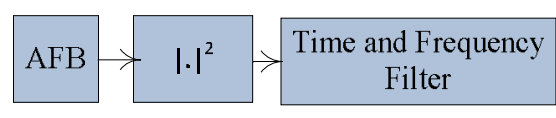

Decision

Device
In the following analysis, it is assumed that the subband sampling rate is equal to the ADC sampling rate divided by the number of FFT/AFB frequency bins. With subband-wise spectrum sensing, the subband signals can be expressed as [3]

$$
Y(m, k)=\left\{\begin{array}{ll}
W(m, k) & H_{0} \\
S(m, k) H_{k}+W(m, k) & H_{1}
\end{array}\right\} .
$$

where $W(m, k)$ is the channel noise, $S(m, k)$ is the transmitted primary user (PU) signal which is seen in subband $k$ of the $m^{\text {th }}$ FFT or AFB output block, and $H_{0}$ and $H_{1}$ denote the absent hypothesis and present hypothesis of a PU, respectively. When only AWGN noise is present, it can be modeled as a zero-mean white Gaussian random variable with variance $\sigma_{w}^{2}$, i.e., $W(m, k)=N\left(0, \sigma_{w}^{2}\right)$. The traditional or enhanced WLAN signals can also be modeled with a zeromean Gaussian variable $S(m, k)=N\left(0, \sigma_{k}^{2}\right)$ and $\sigma_{k}^{2}$ is the variance (power) at subband $k$.

The test statistic, which is calculated as the absolute square of the FFT or AFB output $|Y(m, k)|^{2}$ is compared with a threshold value to decide between $H_{0}$ and $H_{1}$. The threshold is calculated from the noise variance, which is assumed to be known based on previous measurements, and target false alarm probability. Time and/or frequency averaging of the observed $|Y(m, k)|^{2}$ values are necessary to get more reliable decision statistic [3]. Decision statistics at different frequencies can then be calculated as

$$
\tilde{Y}(m, k)=\frac{1}{L_{t} L_{f}} \sum_{l=k-\left\lfloor L_{f} / 2\right]}^{k+\left\lceil L_{f} / 2\right]-1} \sum_{u=m-L_{t}+1}^{m}|Y(u, l)|^{2}
$$

where $L_{f}$ and $L_{t}$ are the filter lengths in frequency and time, respectively. The output of $\tilde{Y}(m, k)$ is passed to threshold function for determining the possible occupancy of the corresponding frequency band at the corresponding time interval. Later on, the sensing time index $m$ is dropped and we use $\tilde{Y}_{k}$ instead of $\tilde{Y}(m, k)$ to simplify the notations.

The probability distribution functions (PDF) of the time filter outputs $\tilde{Y}_{k}$ can be approximated as Gaussian distributions under $H_{0}$ and $H_{1}$ [3]. The threshold value $\lambda$ can be calculated from the target false alarm probability and estimated noise variance. The false alarm probability $P_{F A}$ and detection probability $P_{D}$ can be obtained as follows

$$
\begin{gathered}
P_{D}=\operatorname{Pr}\left(\tilde{Y}_{k}>\lambda \mid H_{1}\right)=Q\left(\frac{\lambda-\left(\sigma_{n}^{2}+\sigma_{k}^{2}\right)}{\sqrt{\left(\sigma_{n}^{2}+\sigma_{k}^{2}\right)^{2} / L_{t} L_{f}}}\right) \\
P_{F A}=\operatorname{Pr}\left(\tilde{Y}_{k}>\lambda \mid H_{0}\right)=Q\left(\frac{\lambda-\sigma_{n}^{2}}{\sqrt{\sigma_{n}^{4} / L_{t} L_{f}}}\right)
\end{gathered}
$$

Figure 3. Scheme of energy detector with AFB and FFT based spectrum analysis 


\section{SPECTRUM UTILIZATION}

Figure 4 shows spectrum utilization process. Proper loading of each subband maximizes the spectrum utilization by a CR. As discussed earlier in the introduction part, rate adaptive loading algorithms are better suited as they offer better control of the interference from a CR to the PU receivers. By fixing the energy constraint to a constant, the rate adaptive loading algorithm maximizes the number of bits per symbol [15]. In the following, $1 / T$ is the symbol rate, $b_{n}$ is the number of bits in subcarrier $n$, and $e_{n}$ is the $n$th subcarrier energy. Then the total number of bits in the available set of $N$ parallel subcarrier symbols is $b=\sum b_{n}$. The overall data rate is $R=b / T$ and the total energy of the $N$ parallel subcarrier symbols is constraint to $\sum_{\sum}^{N} e_{n}=N \bar{e}_{n}$ where $N \bar{e}_{n}$ is the total energy allowed in the systen ${ }^{n=1}$ under consideration.

The largest data rate is achieved by maximizing the sum

$$
b=\frac{1}{2} \sum_{n=1}^{N} \log _{2}\left(\frac{1+e_{n}^{*} g_{n}}{\Gamma}\right)
$$

where $g_{n}=\left|H_{n}\right|^{2} /\left(\sigma_{n}{ }^{2}\right)$ is the subband SNR per unit energy from the transmitter, $\left|H_{n}\right|^{2}$ is the channel gain of the $n$th subband and $\Gamma$ is the gap formulation as given in [15]. $\sigma_{n}{ }^{2}$ is the noise and interference variance in subband $n$, i.e., it contains both the channel white noise and spectrum leakage from the WLAN channels.

The optimum loading can be formulated as

$$
\begin{aligned}
& \max _{e_{n}} b=\frac{1}{2} \sum_{n=1}^{N} \log _{2}\left(\frac{1+e_{n}^{*} g_{n}}{\Gamma}\right) \\
& \text { subject }: N \overline{e_{x}}=\sum_{n=1}^{N} e_{n}
\end{aligned}
$$

The solution to this optimization problem leads to the water filling constant $K$ given below [15].

\section{Spectrum Utilization Algorithm}

The rate maximization algorithm from [15], which is used in this work, is given below

1. Sort the sub-channels based on their gains

$$
g_{1}>g_{2}>g_{3}>\ldots>g_{N}
$$

2. Find the largest $i$ for $e_{N-i}=K-\Gamma / g_{N-i} \leq 0$ with

$$
K=\frac{1}{N-i}\left[N e_{n}+\Gamma \cdot \sum_{n=1}^{N-i} \frac{1}{g_{n}}\right]
$$

3. Eliminate the negative energies $e_{n}<0$ and set $e_{n}=0$ and $b_{n}=0$ for those subcarriers. Set $N^{*}=N-i$, where $i$ is the number of subcarriers with negative energies, $e_{i}=K-\Gamma / g_{i} \leq 0$. The filling constant is also recalculated for the new $N^{*}$.

4. Compute the water-filling energies $e_{n}=K-\Gamma / g_{n}$ where $n=1,2 \cdots, N^{*}$

5. Calculate the data rate for unsorted sub-channels

$$
b_{n}=\log _{2}\left(1+e_{n} \cdot g_{n}\right) / 2 \quad \forall n=1,2, \cdots, N^{*}
$$

The algorithm needs the channel estimate, in the form of subband gains $H_{n}$, as well as estimates of the noise and interference powers $\sigma_{n}{ }^{2}$ of the subbands. The estimates are done using subcarrier wise FFT based energy metric, i.e., using (5) with $L_{f}=1$.

The most important issue in our discussion is the effect of the spectral leakage in OFDM on the throughput of the CR utilizing the spectral hole. Due to the good spectral containment of the subbands in the enhanced OFDM method proposed here, we expect the spectral leakage to be less critical in this case.

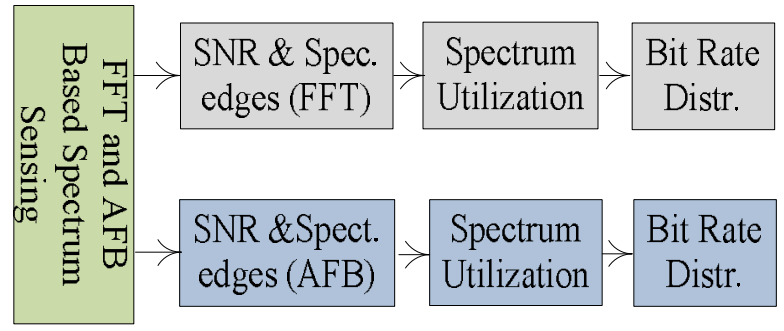

Figure 4. Block diagram of spectrum utilization with water filling after spectrum analysis

\section{SIMULATION RESULTS}

The enhanced WLAN model uses $802.11 \mathrm{~g}$ parameters. However, some modifications are needed to apply the enhanced scheme. Hence, enhanced OFDM appends additional $0.8 \mu$ s to $802.11 \mathrm{~g}$ symbol duration for applying the time windowing. 10 subcarriers per edge are contained in the edge group. In each edge group, the $\mathrm{CP}$ and window duration are $0.4 \mu \mathrm{s}$ and $1.2 \mu \mathrm{s}$, respectively. In inner group, $\mathrm{CP}$ and window durations are $1.2 \mu \mathrm{s}$ and $0.4 \mu \mathrm{s}$, respectively. Regarding the simplified cancellation carrier part, two cancellation carriers and two optimization points are used on each edge. Optimization points are located at the first sidelobes next to cancellation carriers.

In figure 2, we consider a worst-case situation for secondary transmissions using the spectral hole between the two WLAN channels which are at equal power levels. Otherwise the spectral leakage effects would be less critical on the side of the weaker WLAN channel. Traditional and enhanced WLAN systems are based on the 802.11g OFDM standarts. We assume that the spectrum sensing and CR 
transmissions use smaller subchannel bandwidth of $81.5 \mathrm{kHz}$. The time and frequency filtering lengths are chosen as 50 and 5 , respectively, in order to be able to detect other narrowband systems, like Bluetooth [5] in the spectrum sensing part. For the power amplifier, the Rapp model [17] is used with different back-offs. Three cases are considered: no spectral regrowth due to the $\mathrm{PA}$, modest regrowth at the level of -30 $\mathrm{dBr}$ and worst-case regrowth at $-20 \mathrm{dBr}$.

The number of empty subbands both for traditional and enhanced OFDM based PUs are shown in figure 5 with different levels of spectral regrowth. $P_{F A}=0.1$ is chosen as the target false alarm probability in the scenario. The ITU-R Vehicular A channel model is applied for the PU signals. Enhanced OFDM based PU's would allow a clearly higher number of subchannels to be used by the CR system compared to traditional OFDM-based WLAN, especially in the noregrowth and modest regrowth cases. Morever, AFB finds higher number of empty subbands compared to FFT, in reliable way due to the sharp subchannel filtering. With the worst-case regrowth allowed by $802.11 \mathrm{~g}$, these differences dissappear.

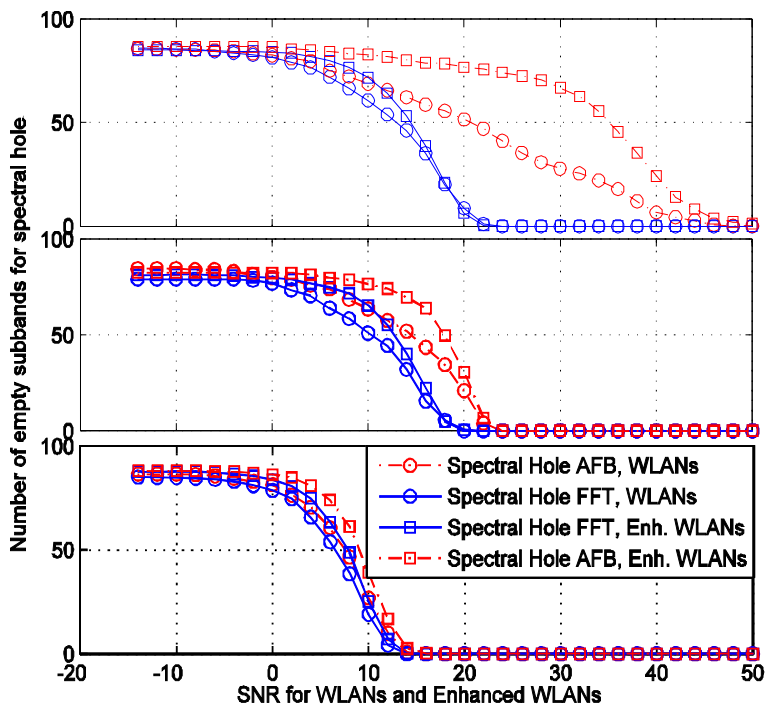

Figure 5. Number of empty subbands in the spectral hole between traditional and enhanced WLANs with target $P_{F A}=0.1$, time record length of 50 , sensing bandwidth of 5 subbands for (a) no spectrum regrowth, (b) modest-case spectrum regrowth, and (c) worst-case spectrum regrowth

The actual false alarm probabilities versus the active primary systems' SNR are illustrated in figure 6. This is actually the probability that a group of 5 subchannels in the center of the gap would be detected to be occupied due to spectral leakage.

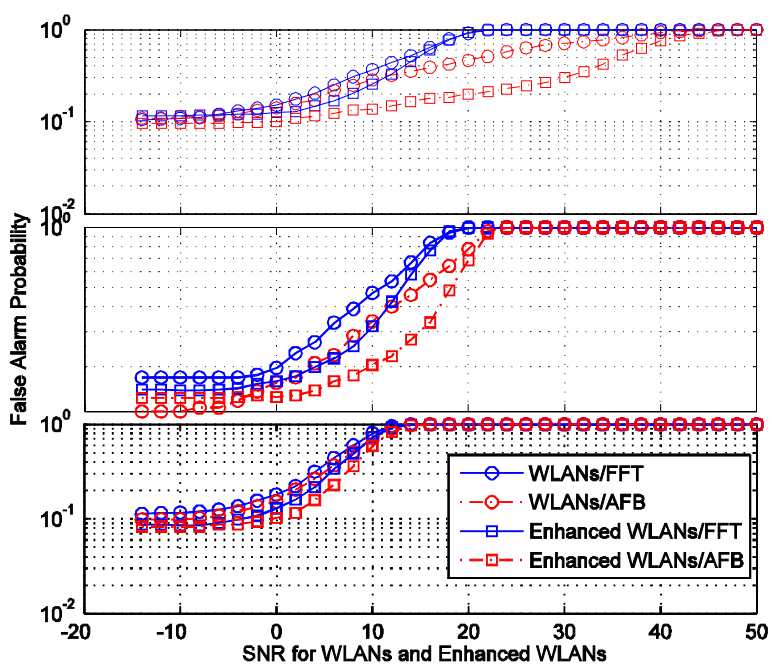

Figure 6. Actual false alarm probability with target $P_{F A}=0.1$ with (a) no spectrum regrowth, (b) modest-case spectrum regrowth, and (c) worst-case spectrum regrowth

The achievable data rate in the spectral hole between two active primary channels is seen in figure 7 . The model gives the theoretical maximum data rate, assuming ideal multicarrier operation for the secondary user (SU) transmission. The subband-wise signal to interference plus noise ratio (SINR) estimates are obtained using time filtering length of 50 samples.

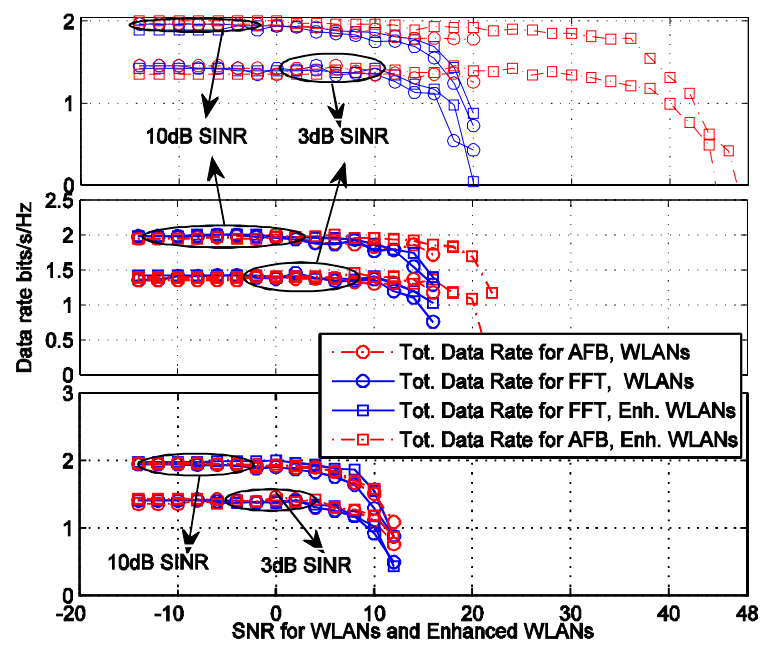

Figure 7. Available data rate as a function of the PU SNR with $10 \mathrm{~dB}$ and 3 $\mathrm{dB}$ SNR for the CR with (a) no spectrum regrowth, (b) modest-case spectrum regrowth, and (c) worst-case spectrum regrowth.

It can be seen that under the high SNR case, the number of subbands that can be used by the CR reduces in the traditional OFDM case due to the spectral leakage. An enhanced OFDM based primary with AFB based spectrum sensing at the $\mathrm{CR}$ would maximize the $\mathrm{CR}$ system performance, while AFB based sensing in the traditional OFDM case shows significant benefit with low or modest spectral regrowth. 
The benefits of enhanced OFDM based WLAN and AFB disappear under the worst case spectral regrowth. It can also be seen that with the used parameters, the spectrum sensing algorithm and the rate adaptive bit loading algorithm (which can be applied after the spectrum has first been detected to be available) end up in using about the same number of sub bands.

\section{CONCLUSION}

The performance of energy detection based spectrum sensing techniques using either FFT or filter bank based spectrum analysis methods for both traditional and enhanced OFDM based WLAN signal models were considered in this study. Furthermore, utilizing the detected spectral holes with water filling algorithms were analyzed in both scenarios.

The enhanced OFDM waveforms provide effective suppression to the spectral sidelobes, which makes it possible for opportunistic users to make use of the nearby frequencies, enhancing the effeciency of the overall spectrum usage. However, a prerequisite for this is improved linearity of the transmitter power amplifier to reduce the spectral regrowth effects. Naturally, the same approach allows independent SU systems to operate side-by-side with minimized guardbands.

The used simplistic SU system model gives the theoretical maximum data rate, assuming ideal multicarrier operation for the SU transmission. This model is justified, e.g., for scenarios where the SU system is used for low-power short range communication with power levels well below the WLANs at reasonable distances from the operating SUs. In this case it can be safely assumed that the SU's don't introduce interference significantly harming the PU operation, and the exact SU system model is not critical. More generally, also the interference introduced by the SU's towards the primary receivers should be taken into consideration in the power allocation context. This is one important topic for future studies.

In practice, also the SU's are preferred to use waveforms with well-contained spectrum, like enhanced OFDM or filter bank based multicarrier (FBMC) [14]. In general, the enhanced OFDM techniques result in certain losses of the spectral efficiency, e.g., due to increased guard interval length in case of time-domain windowing methods. FBMC schemes provide spectrally well-contained waveforms with minimimum overheads from the spectrum efficiency point of view. However, an important benefit of enhanced OFDM schemes, in comparison to FBMC, is high level of compatibility with the existing OFDM based systems. In fact, while the proposed spectrum sensing and allocation scheme is completely independent of the actual waveforms of the primaries, it makes it possible for the opportunistic users to effectively exploit the enhanced spectral containment of the on-going transmissions.

\section{ACKNOWLEDGMENT}

This work was partially supported by Tekniikan Edistamissaation (TES), FP7-ICT project EMPhAtiC under grant no.318362, GETA Graduate School and the Finnish Funding Agency for Technology and Innovation (Tekes) under the project "Enabling Methods for Dynamic Spectrum Access and Cognitive Radio (ENCOR2)" in the TRIAL Program.

\section{REFERENCES}

[1] I. Mitola, J. and J. Maguire, G. Q., "Cognitive radio: making software radios more personal," IEEE Personal Commun. Mag., vol. 6, no. 4, pp. 13-18, Aug. 1999.

[2] Y. Zeng, Y.C. Liang, A. T. Hoang, and R. Zhang, "A Review on Spectrum Sensing for Cognitive Radio: Challenges and Solutions," EURASIP Journal. on Advances. in Sig. Proc, vol. 2010, pp. 1-15, Jan. 2010.

[3] T. Yucek and H. Arslan, "A survey of spectrum sensing algorithms for cognitive radio applications," IEEE Communications Surveys \& Tutorials, vol. 11, no. 1, pp. 116130, March 2009.

[4] S. Dikmese, M. Renfors and H. Dincer, "FFT and Filter Bank Based Spectrum Sensing for WLAN Signals," in Proc. ECCTD2011 conf., Linkoping, Sweden, August 2011.

[5] S. Dikmese and M. Renfors, "Optimized FFT and Filter Bank Based Spectrum Sensing for Bluetooth Signal" in Proc. Wireless Communications and Networking Conference (WCNC 2012), Paris, France.

[6] S. M. Mishra, A. Sahai, and R. W. Broderson, "Cooperative sensing among cognitive radios," in Proc. ICC, Istanbul, Turkey, Jun.11-15, 2006.

[7] T. Weiss, J. Hillenbrand, A. Krohn, and F. Jondral, "Mutual interference in OFDM-based spectrum pooling systems" in Proc. (VTC 2004), May 2004.

[8] A. Sahin and H. Arslan, "Edge Windowing for OFDM Based Systems" Communications Letters, IEEE, vol. 15, no. 11, pp. 1208-1211, 2011.

[9] S. Brandes, I. Cosovic, and M. Schnell, "Sidelobe suppression in OFDM systems by insertion of cancellation carriers" in Proc. (VTC 2005), September, 2005

[10] A. Loulou and M. Renfors, "Effective Schemes for OFDM Sidelobe Control in Fragmented Spectrum Use" in Proc. (PIMRC 2013, London, UK, Sep. 2013.

[11] A. Loulou, S. Afrasiabi Gorgani, and M. Renfors, "Enhanced OFDM Techniques for Fragmented Spectrum Use" in Future Netw., Lisbon. Portugal, July, 2013.

[12] J. Cioffi, "Digital Communication: Signal Processing" at Standford University, Standford, California, USA, 2000.

[13] S. Dikmese, S. Srinivasan and M. Renfors, "FFT and Filter Bank Based Spectrum Sensing and Spectrum Utilization for Cognitive Radios" in Proc. (ISCCSP 2012), Rome, Italy, May 2012.

[14] S. Srinivasan, S. Dikmese and M. Renfors, "Spectrum Sensing and Spectrum Utilization Model for OFDM and FBMC Based Cognitive Radios" in Proc. (SPAWC 2012), Izmir, Turkey, June 2012.

[15] K. Baamrani, A. Ouahmana, and S. Allakib, "Rate adaptive resource allocation for OFDM downlink transmission" AEU Int. Jour. of Elec.and Comm.Vo. 61, Issue 1, 2 Jan. 2007.

[16] G. H. Golub and C. F. Van Loan, "Matrix computations (3rd ed.)". The Johns Hopkins University Press, 1996.

[17] C.Rapp "Effects of the HPA nonlinearity on 4-DPSK OFDM signal for a digital sound broadcasting system" in Proc.Conf. Rec. ECSC'91 Luettich Oct 1991. 